\title{
Design of a Biologically Inspired Humanoid Neck
}

\author{
Steve Barker ${ }^{1}$, Member, IEEE, Luis A. Fuente ${ }^{1}$, Member, IEEE, Khaled Hayatleh ${ }^{1}$, Senior Member, IEEE, \\ Neil Fellows ${ }^{1}$, Jochen J. Steil ${ }^{2}$, Member, IEEE, and Nigel T. Crook ${ }^{1}$, Member, IEEE
}

\begin{abstract}
This paper presents the design of a novel anthropomorphic robotic neck. It mimics the range of movements found in the human neck, actuated by pneumatic artificial muscles. The proposed humanoid neck simulates the anatomical functionality and structure of a human neck. Specifications are made according to biological, anatomical and behavioural data. The preliminary results show that the proposed humanoid neck is able to deliver the range of movements and head velocities comparable to those observedAutomation, 2006. ICRA 2006. Proceedings 2006 IEEE International Conference on. IEEE, 2006. in human necks. These results also demonstrate that biological inspired musculoskeletal robotic systems represent a reliable and robust platform to investigate motion development.
\end{abstract}

\section{INTRODUCTION}

Robots are becoming increasingly pervasive in society. Rather than being confined to precisely defined contexts or hazardous environments, robotic systems are now more common in public and social spaces where an ability to communicate with humans is required. It is well known that non-verbal communication (mostly exhibited by the top part of the human body) is a key feature in human-tohuman communication. Developing robots capable of effectively communicating using non-verbal methods is a complex problem, and perhaps designing a humanoid neck capable of operating in a human-like manner is a challenging task. Head gestures have a more significant impact on emotional acceptability and human-likeness than facial expressions [1].

Recent advances in humanoid robots also highlight the importance of human embodiment in triggering human cognitive processes, increasing the interests in developing musculoskeletal humanoids that simulate the structure of the human body in detail. Previous work in this area includes, for example Kenshiro, a musculoskeletal humanoid with a thoracic spine made of five lumbar vertebrae attached together using a deformed metal spring between each vertebra [2]. The spine is actuated using 10 planar muscles whose location corresponds to the main abdominal human muscles. Mizuuchi et al. developed the humanoid Kotaro [3] which a spine structure formed by five vertebrae, each with 4 point for attaching muscle-tendom (two at the front and two at the back). The muscles are chemical fibers driven by DC motors.

\footnotetext{
${ }^{1}$ Steve Barker, Luis Fuente, Khaled Hayatleh, Neils Fellow and Nigel Crook are with Department of Computing and Communication Technologies, Oxford Brookes University, OX3 0BP Oxford, England. \{stevebarke, lfuente-fernandez, khayatleh, nafellows, ncrook\}@brookes.ac.uk

2 Jochen Steil is with the Research Institute for Cognition and Robotics, Bielefeld University, D-33615 Bielefeld, Germany jsteilecor-lab.uni.bielefeld.de
}

Cla is a human-form flexible-spine robot and its spine is actuated by eight tension-controllable tendons paired in an antagonist manner [4]. Ogawa et al. developed Pneumat-BS [5], a humanoid robot that has a one-vertebra spine actuated by four pneumatic muscles in humanlike arrangements. From the same standpoint, our paper reports the development of a novel 8-DOF musculoskeletal humanoid neck (see Figure 1). Its topology consists of four interconnected wooden vertebrae using ball-and-socket joints and anatomically simulates the structure of the kinematic segments found in the human neck. Its actuation is biologically motivated by the activity of the major muscles governing the motion of necks in humans and relies on eight custom-made McKibben pneumatic artificial muscles (PAMs) whose arrangement closely models the human muscular system.

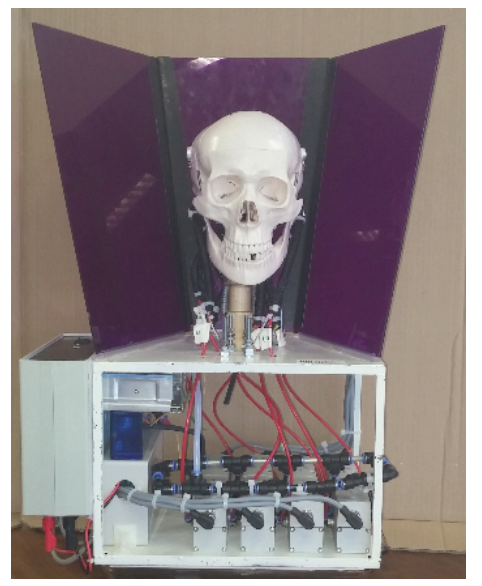

Fig. 1: The humanoid neck "Eddie".

Increasing dexterity and bio-mimetic accuracy in movement are desirable characteristics of musculoskeletal humanoid robots when delicate operations need to be carry out in close proximity to humans. Whilst conventional hydraulic, electric or geared actuator are unable to accurately model them, these are tasks at which pneumatic artificial muscles (PAMs) are evidently good at. PAMs are contractile devices operated by pressurised air [6]. When inflated, they bulge and shorten, and therefore generate a one-directional extensional force. PAMs are usually paired following an antagonist setup in order to generate a restoring movement. Although this kind of coupling is normally avoided by conventional actuators, it presents several benefits in the design of anthropomorphic robots [7], such as flexibility, coordination in the mechanical linkage of joints and compliant behaviour. The proposed designs takes advantage of this natural arrangement 
to generate full-neck simple movements comparable to with the range of motion observe in the human neck.

The rest of the paper is organised as follows: Section II introduces the biological inspiration behind the proposed neck design, Section III describes the mechanical design and hardware specifications, Section IV presents the initial results and discuses their biological plausibility and Section V draws conclusions.

\section{BIOLOGICAL SPECIFICATIONS}

The human neck is the musculoskeletal system connecting the skull with the thoracic spine. It comprises 20 muscles and 10 bones. The section of the spine at the neck is called the cervical spine. The neck exhibits three main types of movement: lateral flexion, dorsal and ventral flexion and lateral rotation.

\section{A. Kinematics of the Cervical Spine}

A human cervical spine structure consists of 7 cervical vertebrae (typically referred as $\mathrm{C} 1$ to $\mathrm{C} 7$ ), however actual controllable kinematic units from the skull to the thoracic spine are the atlas, the axis, the $\mathrm{C} 2-3$ joint and lower cervical spine [8] (see Figure 2). The vertebrae in the cervical spine cannot be controlled independently of each other.

The atlas $(\mathrm{C} 1)$ is the most superior vertebra of the cervical spine and supports the skull. The union between the skull and atlas only permits flexion and extension movements (i.e nodding), otherwise the skull and the atlas move and function essentially as one unit. The axis (C2) is the second cervical vertebra. Its principal function is to provide the pivot upon which the atlas (and therefore the head) rotates sideto-side. Vertebrae $\mathrm{C} 1$ and $\mathrm{C} 2$ constitute the upper cervical spine and are responsible for most of the rotation of the neck. The $C 2-3$ joint is a transitional joint linking the upper cervical spine with the lower cervical spine and therefore, must accommodate the varying demands of the neck above and below it [9]. The lower cervical spine groups C3-C7 vertebrae. These have common morphological and kinematic features and are stacked on one another, separated by intervertebral discs. Each one of these vertebrae has three axis of rotation, two active and one passive. The active axis permits flexion/extension and lateral flextion, and the passive axis (yaw) does not move unless lateral flexion takes place.

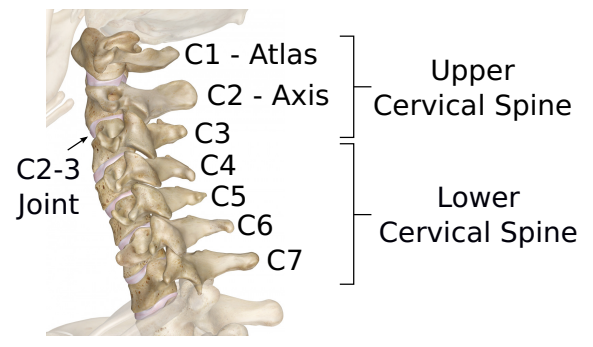

Fig. 2: Anatomical representation of a human neck illustrating its four major kinematic units: Atlas (C1), Axis (C2), $\mathrm{C} 3$ vertebra and its $\mathrm{C} 2-3$ joint and the Lower Cervical Spine (C4-C7). The image of cervical spine is taken form [10].

\section{B. Motion of the Cervical Spine}

This work only considers four different pairs of muscles in the sign of the robotic neck (see Figure 3). Biological studies have shown that these pairs of muscles are the primary drivers of head motion [11], [12] and they are therefore sufficient to replicate the complexity of the human neck and emulate its normal range of motions. Neck muscles normally operate in antagonistic pairs located at opposite sides of the cervical vertebrae. These muscles are:

- The Splenius Capitis muscles which are located at the back of the neck (see Figure 3(a)). They connect the base of the scull with the upper thorax causing the head to rotate and bend towards either side. These muscles are antagonist to Sternocleidomostoid muscles in the rotation of the head.

- The Longissimus Capitis muscles which are at both back sides of the neck (see Figure 3(b)). They originates from the superior thoracic vertebrae and are attached to the mastoid process bone. This extends the head and laterally flexes and rotates by the same amount. This pair of muscles are antagonist to Longus Colli muscles in the flexion of the head.

- The Sternocleidomostoid muscles which are located at the front side of the neck and connect the mastoid bone with the sternum and clavicle (see Figure 3(c)). They are responsible for the rotation of the head and neck.

- The Longus Colli muscles which are situated in the frontal side of the neck, between the Atlas and the upper-most thoracic vertebrae (see Figure 3(d)). They are responsible for the cervical flexion, ipsilateral side flexion and cervical rotation. These muscles also have a postural function.

\section{MECHANICAL DESIGN}

Mizuuchi, Ikuo, et al. "Development of musculoskeletal humanoid kotaro." Robotics and Automation, 2006. ICRA 2006. Proceedings 2006 IEEE International Conference on. IEEE, 2006. The mechanical design of the humanoid neck is divided in four major parts: spine mechanism, muscular mechanism, sensory system and electronic control system. The design and construction of the neck was also guided by the biological specifications described previously in Section II together with the following desirable criteria:

- Capacity to reproduce speeds and displacements of human neck.

- Simplified structure to reduce complexity whilst maintaining biological plausibility.

- Reliability and robustness.

- Where possible, use of standard mechanical components to facilitate the construction of the humanoid neck.

- Modularity to ease the inclusion of additional components.

The following sections describes the different mechanical units of the proposed head. 


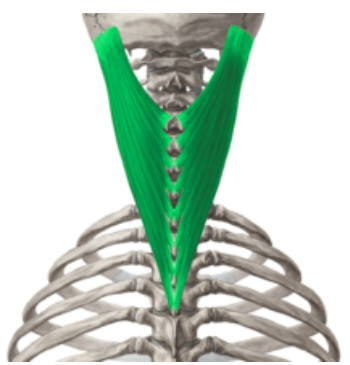

(a) Splenius Capitis

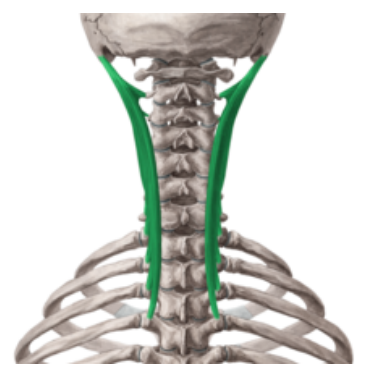

(b) Longissimus Capitis

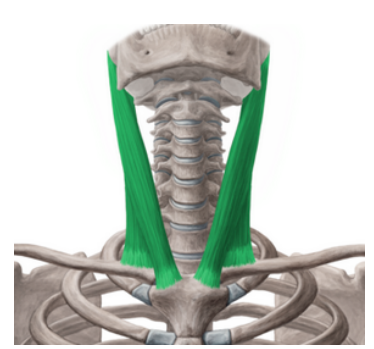

(c) Sternocleidomostoid

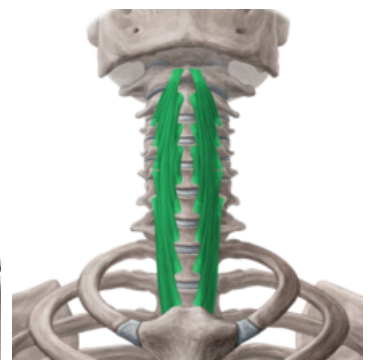

(d) Longus Colli
Fig. 3: Human neck muscles that inspired the design of Eddie (images are courtesy of Kenhub, an online human anatomy atlas [13]).

\section{A. Cervical Spine}

Eddie's cervical spine consists of four stacked and interconnected wooden vertebrae. They are connected using ball-and-socket joints (a compact 3-DOF joint). The uppermost vertebrae imitates the Atlas bone. It has a disk shape and is firmly attached to the plastic skull. Its other end has a concave socket which cradles the second vertebra. The second and third vertebra have a similar cylindrical design with a superior concave upwards surface and a larger cylindrical base with also a concave upwards surface. These vertebrae represent the Axis and C3 vertebra respectively. The last wooden vertebrae represents the $\mathrm{C} 4-7$ vertebrae. It has a longer shape, its superior end has a concave upwards cradles the wooden $\mathrm{C} 3$ and its bottom end is attached to a rectangular-shaped surface which acts as the upper thorax. The wooden vertebrae C3 and C4-7 constitute the upper cervical spine in the proposed design. The length of the designed cervical spine is $13.5 \mathrm{~cm}$ which approximately matches the average length of the cervical part of the spine in a male $(\sim 12.8 \mathrm{~cm})$. All vertebrae have a central hole for an extensible support to pass through them and link them together, whist allowing movement. Each pair of vertebraes is also connected by four steel springs separated $90^{\circ}$ apart. This mimics the action of the intertrasverse and interspinous ligaments, limiting the flexion of the humanoid neck but preserving its flexibility. When powered, the proposed skeletal neck architecture enables the skull to be bent forward (pitch) and laterally (roll) and side-to-side rotated (yaw). It also permits the simulation of other sorts of movements such as the lateral and frontal extension (head upwards) and flexion (head forwards) of the head.

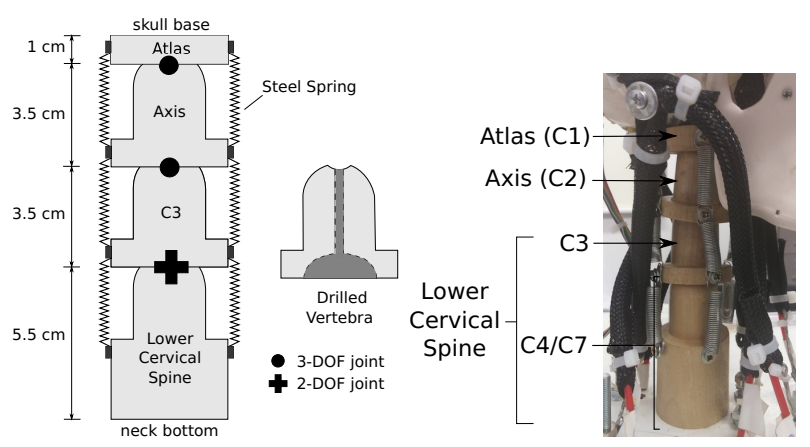

(a)

(b)

Fig. 4: Cervical spine actuation system of Eddie. (a) Two dimensional scheme of the cervical spine actuation system of Eddie. (b) Designed cervical spine and representation of the four major kinematic mechanism in human necks using wooden vertebrae. Vertebrae are connected using 3DOF ball-and-socket joints, with the notable exception of the junction between $\mathrm{C} 3$ vertebra and $\mathrm{C} 4-7$ vertebra which is modelled as 2-DOF joint. This intends to address the lack of rotation in the yaw axis observed amongst vertebrae in the lower cervical spine.

\section{B. Bio-inspired Neck}

The muscular system of the humanoid neck is powered using eight custom-made standard McKibben pneumatic actuators (see Figure 9). Each consists of an internal bladder surrounded by a braided mesh with nylon fibre that is flexible and non-extensive. One end of the bladder is closed and a tube for the air supply is attached to the other end. The lower-end of each actuator is connected to a wingnut-based extensor mechanism located on the surface of the rectangular-shaped upper thorax, while the upper-end is connected to the plastic skull using brackets. Both the wingnuts and brackets hold the air muscles in position within the neck and were designed to resist the reaction force of the PAMs. The position of each of the PAMs corresponds closely to the arrangement of the human muscles described in Section II. The actuators pull the plastic skull in an antagonistic manner in order to produce the desired head movement. The length of the PAMs and neutral position of the head can be tailored by manually adjusting the extensor mechanism.

The humanoid neck is actuated using proportional Festo VPPM air regulators. Unlike traditional two-state switching regulators which only provide an on/off service, proportional air regulators allow the continuous variation of the air flow via an analog voltage. This reduces lunge and undesired shock movements. Festo air regulators are compact and provide a maximum pressure of 10 bars that is comfortably beyond the requirements for this platform. Their operability has been bounded by software to the range of 2-6 bars (safe limit, lower than hardware limit to prevent structural damage) which suffices to produce the patterns of motion 


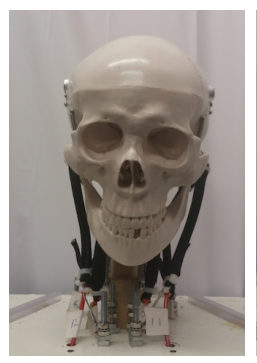

(a)

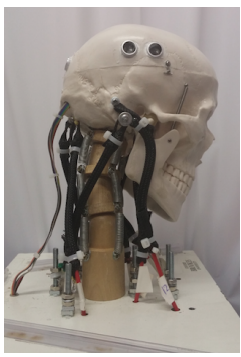

(b)

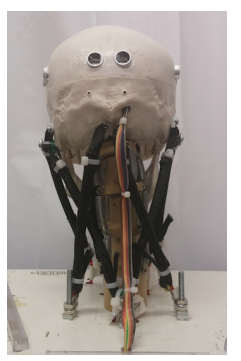

(c)
Fig. 5: Frontal (a), Lateral (b) and Anterior (c) perspectives of Eddie. PAMs are arranged like the human neck muscles highlighted in Figure 3.

found in natural human necks. The technical specifications of the proposed humanoid neck are summarised in Table I.

TABLE I: Hardware specifications of Eddie

\begin{tabular}{c|l}
\hline Neck Height & $13.5 \mathrm{~cm}$ \\
\hline $\begin{array}{c}\text { Neck/Head } \\
\text { Weight }\end{array}$ & $3.5 \mathrm{~kg}$ \\
\hline DOF & 3 \\
\hline $\begin{array}{c}\text { Muscles } \\
\text { Actuators) }\end{array}$ & $\begin{array}{l}\text { Total 8: } \\
\text { 2 Splenius Capitis PAMs }(\sim 170 \mathrm{~mm}) \\
\text { 2 Longissimus Capitis PAMs }(\sim 170 \mathrm{~mm}) \\
\text { 2 Sternocleidomostoid PAMs }(\sim 170 \mathrm{~mm})\end{array}$ \\
\hline 2 Longus Colli PAMS $(\sim 150 \mathrm{~mm})$
\end{tabular}

\section{Sensory System}

The proposed humanoid neck exhibits some distinctive features such as the design of its components and the neck layout which forms an anatomically accurate representation of the human neck. However, its spatial topology also exhibits many practical challenges mainly related to the development of its kinematic model and a robust positioning controller. To address these issues, the proposed neck is endowed with three standard ultrasonic sensors HC-SR04, one 6-DOF gyroscope MPU6050 (see Figure 6), 8 pressure transmitters and a plastic collar (see Figure 1). The ultrasonic sensors are located at the back and on each side of the plastic skull, separated by $90^{\circ}$. The gyroscope is placed at the head dead centre under the skull. The pressure transmitters are located in the Festo regulators. They provide real-time information of the flow pressure and regulate it to compensate air fluctuations. The collar surrounds the plastic skull laterally and from behind and is designed to echo the ultrasonic signals. This configuration provides a nonredundant relative position for every displacement of the head [14] and therefore allows the complete parametrisation of the head motion.
The collar is made of three plastic surfaces, each sloped $20^{\circ}$ vertically and $10^{\circ}$ horizontally. It is sloped to enable orientability [15] because perpendicular surfaces do not allow you to calculate the direction the head is pointing to. The sloped values have been determined experimentally and are sufficient to detect the accurate positioning of the robotic head. The collar is separated $10 \mathrm{~cm}$ laterally and $15 \mathrm{~cm}$ from behind the skull. It is important to note that the collar was uniquely used during the training phase of Eddie's control system. A complete description of Eddie's contro algorithm can be found in Steve et al. (in preparation).

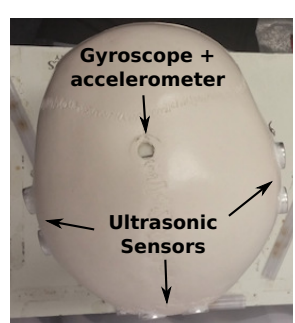

(a)

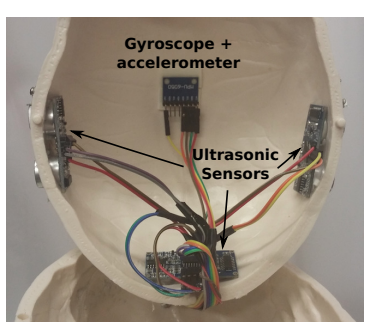

(b)
Fig. 6: Superior (a) and inferior (b) views of the sensors in Eddie's plastic skull

\section{Main Controller}

The humanoid neck is controlled by the commercial Arudino Mega2650 16MHz microcontroller, which is able to drive up to 15 actuators by generating independent Pulse Width Modulation (PWM) signals. These PWM signals are then passed through an active low pass filter, which convert them to true analog signals in the range $0 \mathrm{v}$ to $10 \mathrm{v}$. This range allows control of the Festo proportional valves over their full 0 to 10 bars pressure range. The accelerometer/gyroscope is connected to the Arduino using a $\mathrm{I}^{2} \mathrm{C}$, whilst the ultrasonic sensors are connected directly to the digital input/output pins. Figure 7 provides a block diagram illustrating the overall controller system of the humanoid neck.

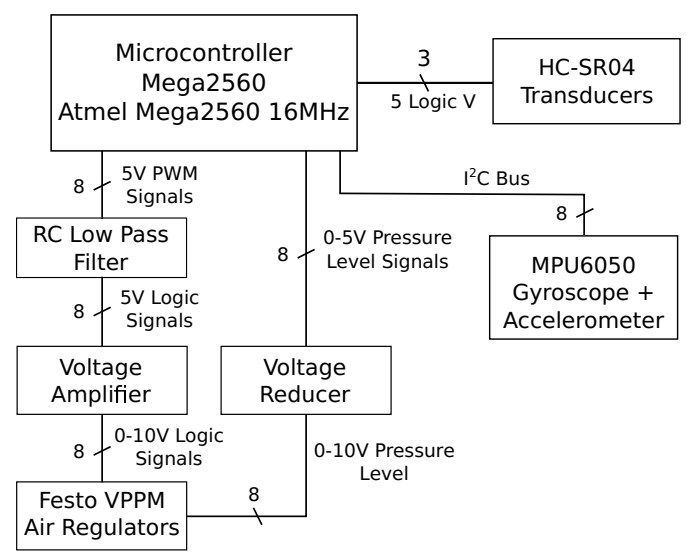

Fig. 7: Block diagram of the circuit used to control the humanoid neck 


\section{PRELIMINARY RESULTS}

\section{A. Biological Plausibility}

Several preliminary tests were made to evaluate the functional specifications of the proposed neck architecture and to determine whether its range of movement meets anatomical boundaries. The data provided by the positional and rotational measures of the gyroscope was used to firstly calibrate the head in an upward motionless resting position and secondly, to track the displacement of the plastic skull. Table II presents the range of movement, velocity and acceleration computed for the three rotation axis of Eddie.

TABLE II: Averaged range of rotation, angular speed and acceleration for the various degrees of freedom of Eddie based on 20 measurements. Antophomorphic data is measured using MPU6050 gyro + accelerometer located on top of the head under the skull and is expressed using the relative $\mathrm{x}$ and $\mathrm{y}$ rotation of the head at its final position with respect to the neural position.

\begin{tabular}{|c|c|c|c|c|c|c|}
\hline \multirow{2}{*}{ Action } & \multicolumn{2}{|c|}{ Angle } & \multicolumn{2}{c|}{ Vel. [m/s] } & \multicolumn{2}{c|}{ Acc. [m/s $\left.{ }^{2}\right]$} \\
\cline { 2 - 7 } & $\mathrm{X}$ & $\mathrm{Y}$ & $\mathrm{X}$ & $\mathrm{Y}$ & $\mathrm{X}$ & $\mathrm{Y}$ \\
\hline Pitch Front & $24.23^{\circ}$ & $3.55^{\circ}$ & 5.76 & 1.56 & 3.17 & 0.08 \\
\hline Pitch Back & $-19.64^{\circ}$ & $-3.43^{\circ}$ & 6.64 & 1.23 & 3.83 & 0.09 \\
\hline Roll Right & $11.69^{\circ}$ & $24.86^{\circ}$ & 3.68 & 4.88 & 1.21 & 3.87 \\
\hline Roll Left & $13.91^{\circ}$ & $-23.08^{\circ}$ & 3.10 & 4.9 & 2.66 & 3.2 \\
\hline Yaw Right & $24.6^{\circ}$ & $0.27^{\circ}$ & 4.57 & 2.57 & 3.39 & 1.9 \\
\hline Yaw Left & $-13.85^{\circ}$ & $-0.65^{\circ}$ & 0.58 & 2.78 & 0.31 & 1.48 \\
\hline
\end{tabular}

The initial results suggest that the proposed neck design is capabwith a minimal rotation around the cervical spinele for producing a range of motion comparable to those observed in human necks. Averaged range of motion for pitch, roll and yaw rotations in elderly humans with ages between 60 and 80 are around $\pm 30^{\circ}, \pm 25^{\circ}$ and $\pm 27^{\circ}$ respectively [17], while the humanoid neck's motion has been bounded by software in the ranges of $\left[-19.63^{\circ}-24.23^{\circ}\right], \pm 24^{\circ}$ and $[-$ $\left.13.85^{\circ}-24.6^{\circ}\right]$ pitch, roll and yaw rotations respectively. However, it is unable to reproduce some of the movements of human necks. As can be seen in Figure 8, the proposed neck exhibits steady dorsal and vental flexion and side-to-side rotation with minimal lateral displacement $( \pm 3.5 \mathrm{y}$-pitch and approx. \pm 0.5 y-yaw) but lateral flexion is inexorably coupled with forward nodding $\left(11.69^{\circ}\right.$ and $13.91^{\circ} \mathrm{x}$-roll). A possible explanation stems from the fact that all PAMs actuators have the same length with the exception of those resenting the Longus Colli muscles and all have the same tension when inflated. While this configuration favours a straight neutral position of the proposed head, the smaller size of the Longus Colli PAMs (front PAMs) induces an undesirable flexion and limits the motion range when the PAMs representing the Longissimus Capitis and Sternonocleidomostoid muscles (lateral PAMs) are gradually inflate and deflate to produce ipsilateral flexion. Overall, these results are encouraging and demonstrate the capacity of the proposed neck design to emulate the range of rotations and motion similar to human necks. Nevertheless, additional adjustments are still required to correct the irregularities observed in the motion of the proposed neck design. Positions of Eddie at its maximum roll and pitch angles are shown in Figure 9.

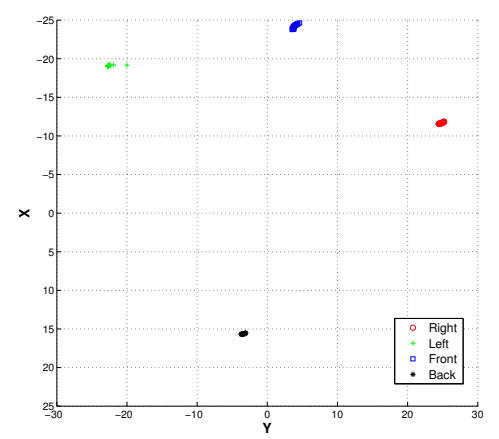

Fig. 8: View from above of the position of the plastic skull in the Cartesian space at Eddie maximum pitch and roll angles for each of the 20 measurements. All points are confined to the same areas in the Cartesian space which indicates the capacity of Eddie to produce precise movements.

\section{B. Control of the Neck}

The structure of the humanoid neck is characterised by 3-DOF. Its motion is actuated by eight PAMs arranged in an antagonist manner and its orientation depends on the rotations of each vertebra in the designed cervical spine. All vertebrae are connected using 3-DOF ball-and-socket joints with the exception of the joint between $\mathrm{C} 3$ and $\mathrm{C} 4-7$ vertebra which is assumed to have only 2-DOF. This mimics the natural movement of human necks in which the rotation of the head mostly occurs in the upper cervical spine [16]. C3-C7 verterbrae act as a natural support and is mostly responsible for the side-to-side bending and flextion/extension movements with a minimal or non-existent rotation. This particular structure makes the humanoid neck redundant because the same neck orientation can be achieved with different positions of the proposed cervical spine and different muscle configurations. The kinematic characteristics of the cervical spine are in Table III. Figure 10 illustrates two different valid configurations of the proposed cervical spine using a stickmodel representation. In a further paper, (Steve et al., in preparation) the relationship the neck kinematic and neck motion will be discussed in detail.

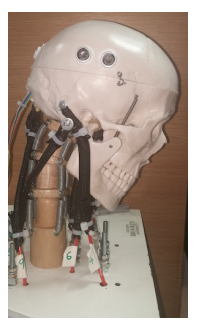

(a)

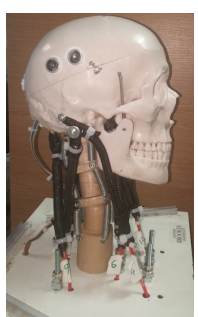

(b)

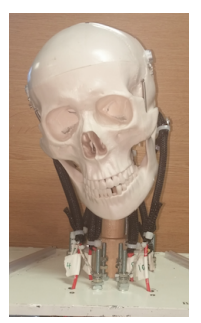

(c)

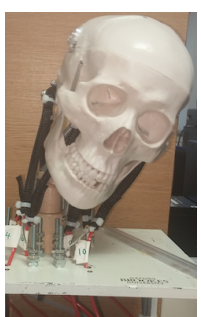

(d)
Fig. 9: Different poses of Eddie neck at its maximum roll and pitch angles as seen in Figure 8. Pitch movements: left pictures. Roll Movements: right pictures. 
TABLE III: DH parameters for Eddie

\begin{tabular}{c|c|c|c|c}
\hline Frame & $a_{i}$ & $\alpha_{i}$ & $d_{i}$ & $\theta_{i}$ \\
\hline \hline 0 & 0 & $\pi / 2$ & $35 \mathrm{~mm}$ & 0 \\
\hline 1 & 0 & $\pi / 2$ & 0 & $\theta_{1}+\pi / 2$ \\
\hline 2 & $25 \mathrm{~mm}$ & $\pi / 2$ & 0 & $\theta_{2}$ \\
\hline 3 & 0 & $\pi / 2$ & 0 & $\theta_{3}$ \\
\hline 4 & 0 & $\pi / 2$ & 0 & $\theta_{4}$ \\
\hline 5 & $25 \mathrm{~mm}$ & $\pi / 2$ & 0 & $\theta_{5}$ \\
\hline 6 & 0 & $\pi / 2$ & 0 & $\theta_{6}$ \\
\hline 7 & 0 & $\pi / 2$ & 0 & $\theta_{7}$ \\
\hline 8 & $110 \mathrm{~mm}$ & $\pi / 2$ & 0 & $\theta_{8}$ \\
\hline \multicolumn{5}{|r}{}
\end{tabular}

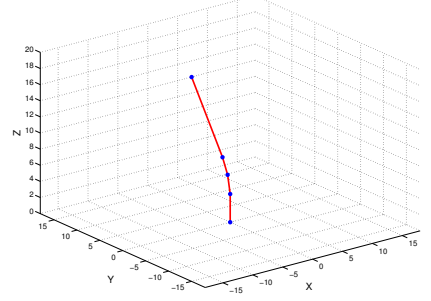

(a)

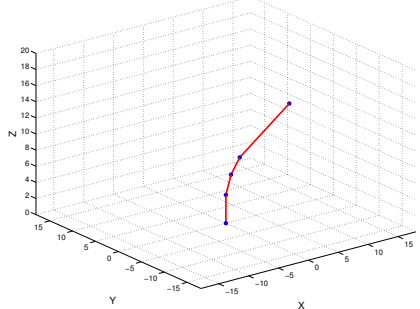

(b)
Fig. 10: Example of two different configurations of the forward kinematic for Eddie's cervical spine.

\section{CONCLUSIONS}

This article has presented the design process of an anthropomorphic neck. The humanoid neck proposes a novel structure highly inspired by the structure and function of the neck musculoskeletal system. The main idea is to have a flexible structure (cervical spine) actuated by surrounding contractile elements (muscles). In the specific implementation, the flexible structure is represented by three interconnected wooden vertebrae plus the Atlas. Individual vertebrae are also linked using four steel springs and an extensible cable which transverses them through a central hole. This coupling contributes to limit the flexion of the cervical spine and provides stability. Its actuation is achieved by eight McKibben air muscles which mimic the action and spatial topology of the principal neck muscles. Furthermore, the spatial topology of the artificial air muscles is biologically inspired by antagonist muscular actuation found in human bodies.

This innovative mechanical design requires the development of appropriate and robust control mechanisms in order to replicate realistic head movements. In this respect, the final design has been complemented with a sensory system composed of three ultrasonic sensors and one gyroscope and eight pressure regulators. The system has been initially used to explore the workspace of the proposed neck system through gathered sensory data. The preliminary results are encouraging and demonstrate that the designed neck is capable of bending roughly within the range of movement of a natural head. Future work will look a the application of Goal Babbling [18] in order to develop a suitable control strategy. This approach has already successfully been applied in the control robot elephant trunk actuated by an array of pneumatic artificial muscles [19]. It involves a trial and error process which enables the controller to discover the set of PAM pressures to accomplish a specific movement. By applying this algorithm to the humanoid neck, it will be possible to a relate precise position to discrete pressure values in each artificial muscle. Last, but not least, work on increasing the sensory capabilities of the humanoid ota11neck is also ongoing.

\section{ACKNOWLEDGMENT}

The authors gratefully acknowledge the contribution of Philips Hughes and Ben Guy for their useful suggestions and advice during the development process.

\section{REFERENCES}

[1] C. Özgen, Canan. "Human-like robot head design" PhD diss., Middle East Technical University, 2007.

[2] Y. Nakanishi, et al."Design concept of detail musculoskeletal humanoid "Kenshiro"-Toward a real human body musculoskeletal simulator." Humanoid Robots (Humanoids), 2012 IEEE-RAS International Conference on, pp. 1-6, IEEE, 2012.

[3] I. Mizuuchi, et al. "Development of musculoskeletal humanoid kotaro." Robotics and Automation, ICRA 2006, IEEE International Conference on., pp. 82-86, 2006.

[4] I. Mizuuchi, M. Inaba, and H. Inoue, "A Flexible Spine HumanForm Robot Development and Control of the Posture of the Spine ", Robotics and Automation, ICRA 2001, IEEE International Conference on. pp. 20992104, 2001.

[5] K. Ogawa, k. Narioka and K. Hosoda. "Development of whole-body humanoid "pneumat-BS" with pneumatic musculoskeletal system". Robotics and Automation 2011, ICRA 1998, IEEE/RSJ International Conference on, pp. 4838-4843, 2011.

[6] F. Daerden and D. Lefeber. "Pneumatic artificial muscles: actuators for robotics and automation," European journal of mechanical and environmental engineering, vol. 47, no. 1, pp. 11-21, 2002.

[7] S. T. Davis and D. G. Caldwell. "The bio-mimetic design of a robot primate using pneumatic muscle actuators" 4th International Conference on Climbing and Walking Robots, pp. 197-204, 2001.

[8] N. Bogduk and S. Mercer. "Biomechanics of the cervical spine. I: Normal kinematics," Clinical Biomechanics vol. 15, no. 9, pp. 633648,2000

[9] C. Liebenson. "The C2/C3 Joint and Neck Pain", Dynamic Chiropractic, vol. 14 , no. 4 (1996).

[10] T. Taylor. "Cervical Vertebrae" InnerBody.com, HowToMedia, Inc., Oct 2012. [Online]. Available: http://www.innerbody.com/anatomy/skeletal/cervical-vertebrae-lateral.

[11] M. B. Dutia. "The muscles and joints of the neck: their specialisation and role in head movement," Progress in neurobiology vol. 37, no. 2, pp. $165-178,2001$

[12] A. R. Tilley. "The measure of man and woman," 1993.

[13] N. Hapke, "Kenhub", accessed 8th February 2014. [Online]. Available: https://www.kenhub.com/

[14] O. Wijk, P. Jensfelt, and H. I. Christensen. "Triangulation based fusion of ultrasonic sensor data," Robotics and Automation 1998, ICRA 1998, IEEE International Conference on, vol. 4, 1998.

[15] N. Hitchin, "Geometry of surfaces", Lecture Notes Oxford University (Oxford), 2004.

[16] J. H. Bland and D. R. Boushey. "Anatomy and physiology of the cervical spine," Seminars in arthritis and rheumatism vol. 20, no. 1, pp. 1-20, 1990

[17] J. W. Youdas, T. R. Garrett, V. J. Suman, C. L. Bogard, H. O. Hallman, and J. R. Carey. "Normal range of motion of the cervical spine: an initial goniometric study," Physical Therapy, vol. 72, no. 11, pp. 770-780, 1992.

[18] M. Rolf, J. J. Steil, and M. Gienger. "Goal babbling permits direct learning of inverse kinematics," Autonomous Mental Development 2010, TAMD 2010, IEEE Transactions on vol.2, no. 3 pp. 216-229, 2010.

[19] M. Rolf and J. J. Steil. "Efficient exploratory learning of inverse kinematics on a bionic elephant trunk," IEEE Trans. Neural Networks and Learning Systems, vol. 25, no. 6, pp. 1147-1160, 2014. 